\title{
Collapsible fan Blade Force Analysis and Energy Transformation Peng Cui
}

\author{
North China Electric Power University, Beijing, 102206
}

alanncepu@foxmail.com

Keywords: Collapsible fan blades; Betz theory; Wind turbines power; Wind speed

\begin{abstract}
A new structure named 'collapsible fan blades' is presented in this paper, which is fixing blades and a spring via metal rods. In this structure, based on Betz theory, the furl of the fan blade is realized by compressing the spring. On the purpose of getting relation between wind speed and power, this paper firstly derived the expression between wind speed, wind force and wind turbine power. Then presented the structure and working principle of the collapsible fan blades. By means of force analysis and moment analysis, an expression on a randomly given wind speed and the spring shrinkage was proposed. In this expression, different wind speeds corresponded to different spring compression, so that the fan blades' angle can be figured out. With this angle, after calculating windward area, the power of wind turbines under different wind speeds can be obtained.
\end{abstract}

\section{Force on Fan Blade and Energy Conversion}

Model Hypothesis. Based on linear momentum theory and Betz theory, we use the method of flow tube analysis to predict the axial thrust and power output of the ideal horizontal axis wind turbine. In Betz theory, the effective scanning area of the blade can be regarded as a disc, which's two sides of the pressure is not equal. So we make the following assumptions:

(1) There is no yaw angle, tilt angle and taper angle of the fan blade, which can be regarded as a flat disc.

(2) Regard the friction resistance of blades.

(3) The model of blade rotation can be simplified to a plurality of unit flow tubes.

(4) The air pressure on the front and back of the blade is equal.

(5) The force acting on the blade is uniform.

(6) Ignore the rotation of the air behind the blade.

(7) The central axis of the blade is in a horizontal position and the wind direction is horizontal.

\section{Parameter Declaration.}

Table 1

\begin{tabular}{|c|l|}
\hline Parameter symbol & Physical meaning \\
\hline$\infty$ & Means far away from the blade \\
\hline$d$ & Indicates the location of the fan blade \\
\hline$w$ & Indicates a smooth airflow area after the fan \\
\hline$F$ & Wind force $(\mathrm{N})$ \\
\hline$P$ & The power absorbed by the fan from the wind $(\mathrm{W})$ \\
\hline$\rho$ & Air density $\left(\mathrm{kg} \cdot \mathrm{m}^{-3}\right)$ \\
\hline$\alpha$ & Fan's area after rotating $\left(\mathrm{m}^{2}\right)$ \\
\hline$p_{d}^{+}$ & Axial wind velocity \\
\hline$p_{d}^{-}$ & The pressure front of fan $(\mathrm{Pa})$ \\
\hline & The pressure back of fan $(\mathrm{Pa})$ \\
\hline
\end{tabular}

Derivation of Wind Power and Absorption Power. Based on the one-dimensional momentum 
theory, we can obtain the relationship between the blade force and air velocity. According to the law of conservation of mass, the air mass per unit time should be kept constant over the swept area of the fan blade

$$
\rho A_{\infty} v_{\infty}=\rho A_{d} v_{d}=\rho A_{w} v_{w}
$$

Let $F$ represent the force of air flow on the fan blade. According to $F=\frac{\mathrm{d}(m v)}{\mathrm{d} t}$,

$$
F=\left(v_{\infty}-v_{w}\right) \rho A_{d} v_{d}
$$

The change of momentum comes from the pressure change on the front and back of the leaves. Which is

$\frac{\mathrm{d}(m v)}{\mathrm{d} t}=\left(p_{d}^{+}-p_{d}^{-}\right) A_{d}$

Thus,

$\left(p_{d}^{+}-p_{d}^{-}\right) A_{d}=\left(v_{\infty}-v_{w}\right) \rho A_{d} v_{d}$

According to Bernoulli Equation $\frac{1}{2} \rho v^{2}+p+\rho g h=$ constant ,in front of the fan blade,

$\frac{1}{2} \rho_{\infty} v_{\infty}^{2}+p_{\infty}+\rho_{\infty} g h_{\infty}=\frac{1}{2} \rho_{d} v_{d}^{2}+p_{d}+\rho_{d} g h_{d}$

According to the assumptions before $\rho_{\infty}=\rho_{d}, h_{\infty}=h_{d}$, so

$\frac{1}{2} \rho v_{\infty}^{2}+p_{\infty}=\frac{1}{2} \rho v_{d}^{2}+p_{d}^{+}$

In the same way, behind the fan blades

$\frac{1}{2} \rho v_{w}^{2}+p_{\infty}=\frac{1}{2} \rho v_{d}^{2}+p_{d}^{-}$

(6) $-(7)$,

$p_{d}^{+}-p_{d}^{-}=\frac{1}{2} \rho\left(v_{\infty}^{2}-v_{w}^{2}\right)$

According to (3).(4).(8)

$\frac{1}{2} \rho\left(v_{\infty}^{2}-v_{w}^{2}\right) A_{d}=\left(v_{\infty}-v_{w}\right) \rho A_{d} v_{d}$

The fan has a reducing effect on the wind, so we can make $v_{d}=(1-\alpha) v_{\infty}$, so the wind force is

$F=2 \rho A_{d} v_{\infty}^{2} \alpha(1-\alpha)$

And the power from the wind to the fan is

$P=F v_{d}=2 \rho A_{d} v_{\infty}^{3} \alpha(1-\alpha)^{2}$

\section{Blade Collapsing}

Process of Blade Collapsing. In collapsible blade structure, the fan blade is linked with the spring, through metal rod. Connection point is fixed by rotatable bearing. Spring baffle is connected with a good-elastic spring, the other end of the spring is fixed with a central axis. When the wind speed is zero, there is no force on the fan, so there is no force on spring, as is shown in Fig.3. When the wind speed is greater than zero, the blades will be contracted to the action of wind, and gradually collapsing. In this process, it presses the spring. Being compressed, according to Newton the third law, the spring will achieve a equilibrium state by pushing a force back. Which is the force balance between the spring force and wind. The structure is shown in Fig.3. With the increasing of wind speed, the wind blades will get more and more force from the spring. By this reaction force, the system comes to a new balance again. 


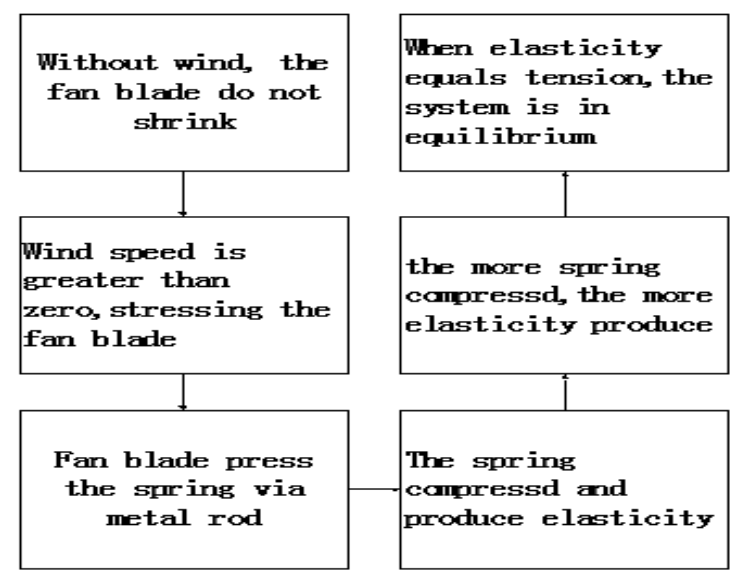

Figure 1. Finite Fan blade shrink process

Model Structure. There is two structures, which correspond to two states, without wind and not.
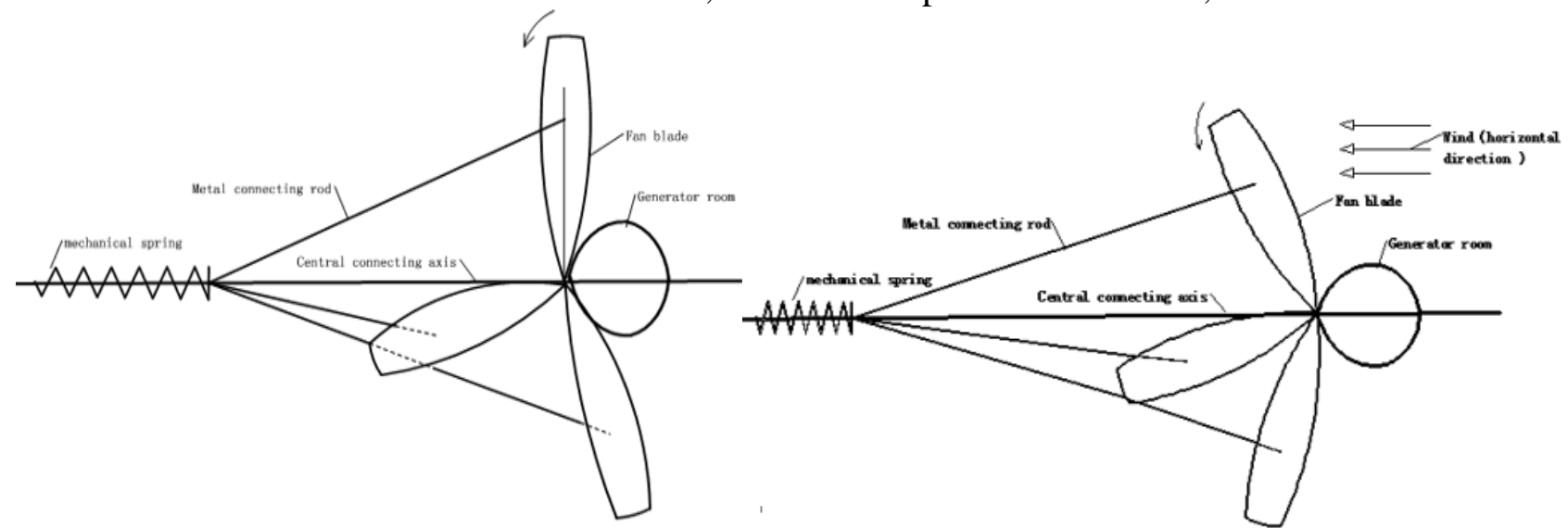

Figure 2. Finite Collapsible fan (in Breezeless status) Figure 3. Collapsible fan(At a certain wind speed)

Tress Analysis of a State after Collapsing

Parameter Description

Table 2

\begin{tabular}{|c|c|}
\hline Parameter symbol & $\begin{array}{r}\text { Physical meaning } \\
\text { In the connection of metal rod and spring, the support force received by } \\
\text { metal rod }(\mathrm{N})\end{array}$ \\
\hline$F$ & Horizontal wind( $\mathrm{N})$ \\
\hline$T$ & spring force $(\mathrm{N})$ \\
\hline$\beta$ & The angle between the fan blade and the vertical axis $\left(^{\circ}\right)$ \\
\hline$F_{x}$ & Horizontal force acting on the metal rod( $)$ \\
\hline$F_{y}$ & Vertical force acting on the metal rod(N) \\
\hline
\end{tabular}




\section{Stress and Moment Analysis}

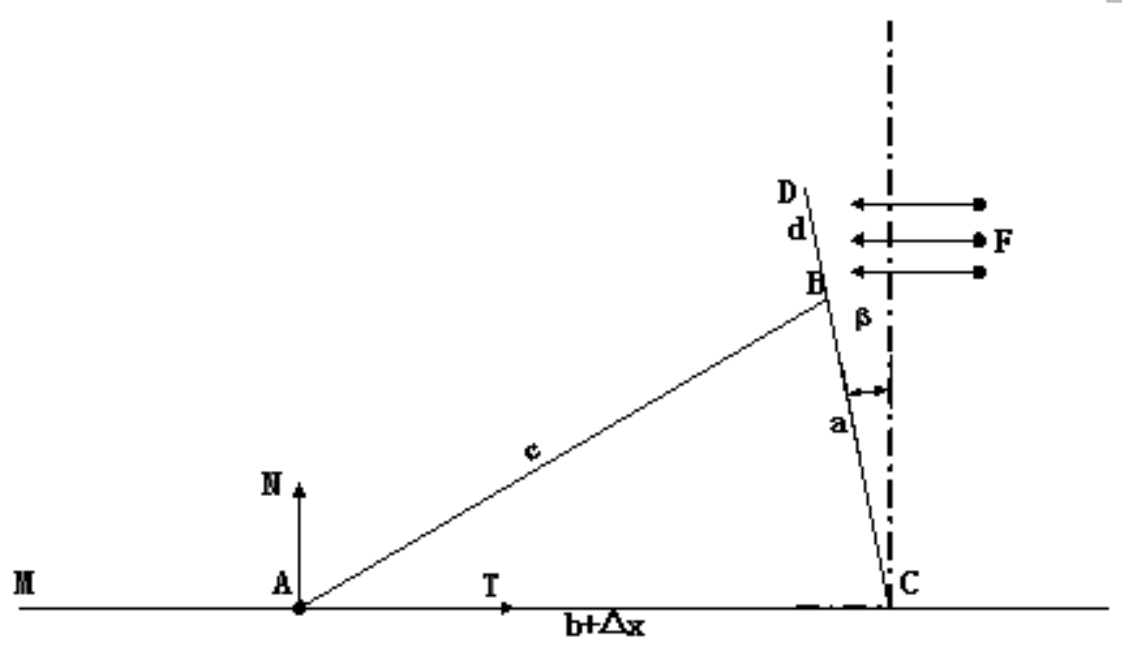

Figure 4. Finite Force analysis in total

Because of the spatial distribution of the three fan blades, we can only consider one blade.

In Fig .4,firstly we analyse the moment as a whole, taking $\mathrm{C}$ as a rotation point:

$N \cdot(b+\Delta x)=F \cos \beta \cdot \frac{a+d}{2}$

Secondly, analyse the force on metal rod in Fig.5:

$F_{x}=T=k \cdot \Delta x$

$F_{y}=N$

By Newton's third law, $\overrightarrow{F_{x}}=-\overrightarrow{F_{x}^{\prime}}, \overrightarrow{F_{y}}=-\overrightarrow{F_{y}^{\prime}}$. Based on that, we analyse the moment on the blade in Fig.6:

$\left(F_{y} \cdot \sin \beta+F_{x} \cdot \cos \beta\right) \cdot a=F \cos \beta \cdot \frac{a+d}{2}$

From (12) (15),we can get:

$(N \cdot \sin \beta+k \Delta x \cdot \cos \beta) \cdot a=F \cos \beta \cdot \frac{a+d}{2}$

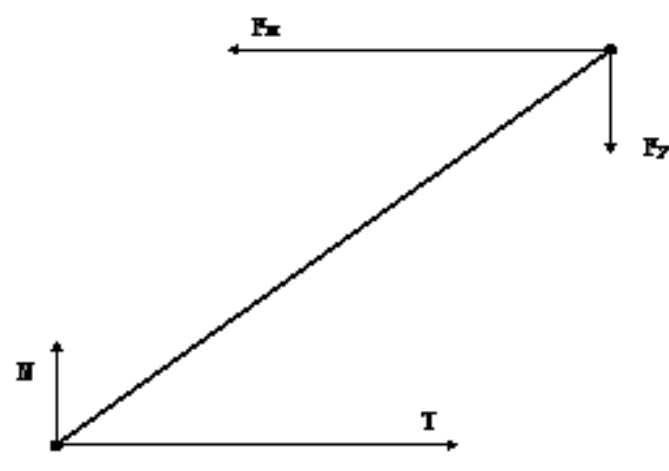

Figure 5. Finite Force analysis about metal rod

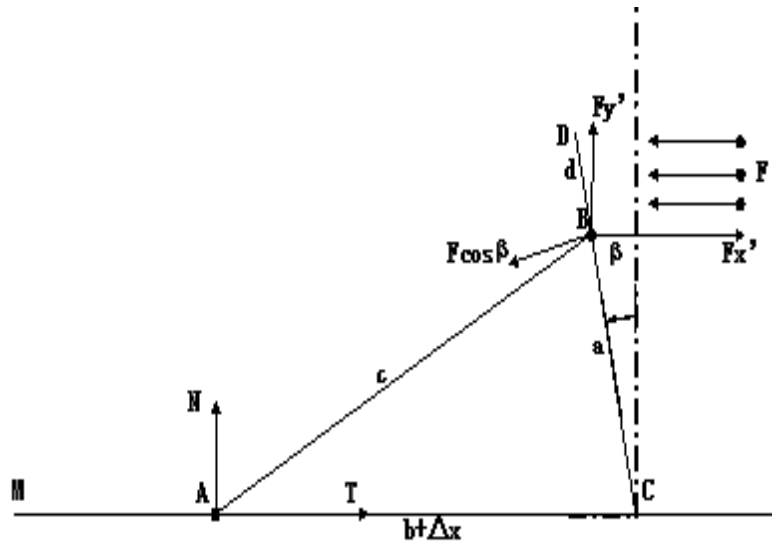

Figure 6. Finite Moment analysis of an blade

\section{Expression of Wind Speed and Spring Contraction}

From the geometric relation in Fig .3, by Cosine theorem, 


$$
\cos \left(90^{\circ}-\beta\right)=\sin (\beta)=\frac{a^{2}+(b+\Delta x)^{2}-c^{2}}{2 a(b+\Delta x)}
$$

By(12) (16), (17)can be simplified:

$$
F=\frac{4 a \cdot k \cdot \Delta x \cdot(b+\Delta x)}{\left[(b+\Delta x)^{2}-a^{2}+c^{2}\right](a+d)}
$$

Take(10)into (18),

$$
\begin{aligned}
& v_{\infty}^{2}=\frac{2 a k \cdot \Delta x \cdot(b+\Delta x)}{\left[(b+\Delta x)^{2}+c^{2}-a^{2}\right] \cdot(a+d) \cdot \rho \cdot A_{d} \cdot \alpha(1-\alpha)}=M_{f a n} \cdot \frac{\Delta x \cdot(b+\Delta x)}{(b+\Delta x)^{2}+c^{2}-a^{2}} \\
& M_{f a n}=\frac{2 a k}{(a+d) \cdot \rho \cdot A_{d} \cdot \alpha(1-\alpha)}
\end{aligned}
$$

In this eqution, $M_{\text {fan }}$ is a constant related to the structure of fan. Once the structure is setted, $M_{\text {fan }}$ will be a definitized constant.

\section{Conclusion}

According to $v_{\infty}^{2}=M_{f a n} \cdot \frac{\Delta x \cdot(b+\Delta x)}{(b+\Delta x)^{2}+c^{2}-a^{2}}$, we take a given wind speed to this equation. After solving the quadratic equation with one unknown, we can calculate the displacement of the spring. Besides, in $\sin (\beta)=\frac{a^{2}+(b+\Delta x)^{2}-c^{2}}{2 a(b+\Delta x)}$, we can find the angle of fan blade as well, which means we can know the windward area $A_{d}$.In this way, the power transmissed by Generator in strong wind can finally be quantitatively expressed.

\section{References:}

[1] The Lanchester-Betz-Joukowsky limit [J]. Gijs A.M. van Kuik. Wiley, 2007.

[2] A numerical analysis to evaluate Betz's Law for vertical axis wind turbines [J]. F Thönnißen; M Marnett; B Roidl; W Schröder; Institute of Physics, 2016.

[3] Wind Energy Conversion Efficiency Limit[J]. Gabriel C. K. Lam. MultiScience, 2009, 431-436.

[4] Classic Bernoulli's principle derivation and its working hypotheses [J]. Edson R Marciotto. Institute of Physics, 2016.

[5] Blade of wind driven generator [M].Xin Cai; Pan Pan; Jie Zhu; Rongrong Gu. Water conservancy and Hydropower Press, 2014, 43-46.

[6] The parametered modeling technology study for the civil aircraft engine fan blade [J]. Haijun Zeng; Youchao Sun. Emerald, 2012, 96-104.

[7] Jowder F A R, Ooi B T. VSC-HVDC station with SSSC characteristics [J]. IEEE Transactions on Power Electronics, 2004, 19(4):1053-1059.

[8] Sørensen J N. Aerodynamic Aspects of Wind Energy Conversion [J]. Annual Review of Fluid Mechanics, 2011, 43(1):427-448.

[9] http://baike.so.com/doc/3923647-4117812.html.

[10] Ashraf M A, Young J, Lai J C S, et al. Numerical Analysis of an Oscillating-Wing Wind and Hydropower Generator[J]. Aiaa Journal, 2011, 49(7):1374-1386. 